\title{
Comparación de dos dietas en la adaptabilidad al cautiverio de neonatos de tortuga golfina en Choluteca, Honduras
}

Comparison of two diets in the adaptability to captivity of neonates of olive ridley turtles in Choluteca, Honduras

DOI 10.5377/rct.v0i24.7874

Edgar Osiris Carranza ${ }^{1}$

Yolany Aguilar, Kenia Rodríguez, Jessy Montoya, Daniel Quiroz, Yelsin Rivas ${ }^{2}$

\section{RESUMEN}

En Honduras desde el año 1974 se realizan acciones para contribuir a preservar la tortuga golfina Lepidochelys olivacea en el Golfo de Fonseca con la instalación de campamentos tortugueros, pero al hacer liberaciones en la fase de neonato la tasa de sobrevivencia es baja. El objetivo consistió en comparar la adaptabilidad, el crecimiento y la sobrevivencia de neonatos alimentados con una dieta comercial para tilapia y otra con carne de corvina durante 35 días en cautiverio. El estudio se realizó en el laboratorio húmedo del CURLP, se recolectaron 12 neonatos de la playa de Punta Ratón con peso promedio de $17 \mathrm{~g}$, estos se distribuyeron en seis recipientes de $0.22 \mathrm{~m} 3$ y mantuvieron con agua marina filtrada, se manejaron como tratamientos la alimentación de carne fresca de corvina y alimento formulado de tilapia al $35 \%$ de proteína cruda durante 35 días, se hicieron tres réplicas de cada tratamiento. Se evaluó la adaptabilidad, el crecimiento en peso y en la longitud curvo de carapacho (LLC). Los neonatos demostraron una adaptación al cautiverio y no fueron influenciados por el tipo de alimento suministrado, de igual forma no se encontraron diferencias estadísticas en los crecimientos semanales y finales en peso y en LCC en los neonatos. Se logró al final del experimento el $100 \%$ de sobrevivencia en los dos tratamientos. La dieta de tilapia puede utilizarse para alimentar a los neonatos de tortuga debido a su composición nutricional y puede ser una opción por la accesibilidad y costo, así como su uso para el crecimiento de los neonatos en cautiverio.

Palabras clave: Neonatos, tortugas, adaptabilidad, longitud curvo del carapacho.

\footnotetext{
${ }^{1}$ Profesor del Departamento de Acuicultura y Biología Marina, Centro Regional Universitario del Litoral Pacífico, UNAH: edgar.carranza@unah.edu.hn

2 Departamento de Acuícultura y Biología Marina, Centro Regional Universitario Litoral Pacífico (CURLP-UNAH)
} 


\section{ABSTRACT}

In Honduras since the year 1974, actions were taken to help preserve the Turtle Ridley Lepidochelys olivacea in the Gulf of Fonseca with the installation of turtle camps, but when releasing in the neonathal phase the survival rate is low. The objective was to compare the adaptability, growth and survival of newborn fed a commercial diet for tilapia and one with meagre meat for 35 days in captivity. The study was carried out in the humid laboratory of the CURLP, were collected 12 newborns of the beach of Punta Raton with average weight of $17 \mathrm{~g}$, these were distributed in six vesseIs of $0.22 \mathrm{~m} 3$ and maintained with filtered seawater, were managed as treatments the feeding of fresh meagre meat and tilapia formulated food to $35 \%$ crude protein for 35 days, three replicas of each treatment were made. Adaptability, weight growth, and curved length of carapacho (LCC) were assessed. The newborns showed an adaptation to captivity and were not influenced by the type of food supplied, likewise no statistical differences were found in the weekly and final growths in weight and LCC in infants. At the end of the experiment, $100 \%$ of survival was achieved in the two treatments. The tilapia diet can be used to feed turtle hatchlings because of their nutritional composition and may be an option for accessibility and cost, as well as its use for the growth of captive infants.

Keywords: Neonates, turtles, adaptability, curved carapace length. 


\section{INTRODUCCIÓN}

La tortuga golfina Lepidochelys olivacea es una de las especies de tortuga marina que tiene una amplia distribución a nivel mundial, principalmente en las aguas tropicales y subtropicales de los océanos Indico, Pacífico y Atlántico Central. En el Pacífico Oriental se encuentra desde California y el Golfo de California hasta la frontera norte de Chile (Castro González, Miranda Becerra, \& Pérez Gil, 2010). Sin embargo, sus poblaciones se han reducido en los sitios de anidamiento debido a la presencia de las actividades antrópicas provocadas por el hombre (Aggarwal, Velavan, Hendre, \& Shanker, 2004). Por lo que provoca un difícil proceso de reproducción y una baja tasa supervivencia.

Esta especie en la actualidad se encuentra en peligro, según los listados de las especies amenazadas de la Unión Internacional para la Conservación de la Naturaleza (IUCN, 2011). Las principales acciones que han afectado las poblaciones de la tortuga golfina han sido el consumo y la comercialización de huevos, carne, aceite y caparazones, así como la degradación de su hábitat, la captura incidental por pesca artesanal e industrial la contaminación de los mares por hidrocarburos, derivados del petróleo y material no biodegradable (Camacho, Amorocho, Mejía, Palacio, \& Rondón, 2008). Sin embargo, en las costas hondureñas, el avistamiento y las poblaciones durante el período de anidamiento se han reducido drásticamente en los últimos 20 años (PNUD, 2019).

Desde el año 1974 se desarrollan acciones para conservar la tortuga golfina a través de la instauración de un periodo de veda que prohíbe el consumo y comercialización de huevos, y el establecimiento de campamentos tortugueros en Honduras. Durante el período de agosto a noviembre de cada año se instalan campamentos en las playas de Punta Ratón, Boca de Rio viejo, Cedeño y el Venado con el propósito de contar con centros de anidamiento artificial que aumentan la tasa de natalidad de los huevos de tortuga y una vez eclosionados a los pocos días son liberados al mar (Mi Ambiente, 2018). No obstante, existe factores que limitan a los neonatos llegar a la etapa de adultez debido a que en sus primeros días de nacidos están más vulnerables a la caza por depredadores naturales, se estima que únicamente un individuo de cada mil llega a ser adulto (IAC/CIT, 2007). Esto expone la fragilidad de la especie en los ecosistemas marinos. Para lograr una mejor tasa de sobrevivencia en los neonatos se sugiere la liberación de animales más desarrollados al tener una mayor tolerancia para sobrevivir al medio natural. Los neonatos que se conservan en cautiverio por más de 30 días cuentan con mayor capacidad de adaptación y mejor sobre- 
vivencia que las tortugas liberadas entre las 24 y 76 horas de eclosionadas (Marquez, 2013). El manejo de estos organismos en cautiverio por un determinado periodo de tiempo podría ser una estrategia de manejo para lograr una mayor conservación de la especie, no obstante, es necesario conocer su conducta alimentaria.

La tortuga golfina desempeña una función significativa en los ecosistemas marinos, su importancia al igual que otras especies de tortugas marinas es que contribuyen en sostener la salud de los océanos. El consumo en abundancia de organismo marinos como cangrejos, camarones, moluscos, bivalvos, y cnidiarias como las medusas, ayudan en mantener las poblaciones adecuadas de estas especies que se reproducen masivamente (Colman, Sampaio, Weber, \& Comin, 2014), también al consumir el pasto de las praderas marinas, aumenta la productividad y el contenido de nutrientes en el pasto. La falta de este pastoreo en las praderas obstruiría las corrientes marinas y se provocaría la descomposición del hábitat (Wilson, Miller, Allison, \& Magliocca, 2006).

Los hábitos alimenticios de la tortuga golfina cambian según su etapa de crecimiento. En las primeras 72 horas después del nacimiento no se alimentan debido a las reservas naturales de nutrientes proveídas por su saco vitelino que contiene alto contenido de proteína y sustancias energéticas como glúcidos y lípidos (Marquez, 2013), luego y durante toda la fase de juvenil desarrollan preferencias carnívoras consumiendo crustáceos, bivalvos y peces pequeños como juveniles de corvina y otras especies (Colman, Sampaio, Weber, \& Comin, 2014), al llegar a la adultez se convierten en omnívoros oportunistas en donde ingieren el alimento que encuentra a su paso, sobre todo el bentos en las áreas arenosas cuando el suministro es escaso (Casas \& Gómez Aguirre, 1980). Por tener hábitos carnívoros en las primeras etapas de vida la tortuga golfina demanda una dieta rica en proteína. Al mantener a los neonatos en cautiverio se deben contar con una dieta que le provea los requerimientos nutricionales que necesitan. Esta fase es la más crítica debido a que son más expuestos a enfermedades, pueden desarrollar un lento crecimiento y observarse mortalidades, la dieta debe contar con proteína de calidad que estimulen la formación de tejidos y masa muscular (Pelegrín, Álvarez, Fraga, \& Galindo, 2003). Es posible que existan productos sustitutos a un menor costo que el alimento natural y que cumpla con las necesidades nutricionales.

Una alternativa podría ser el uso de alimento balanceado de tilapia con alto contenido de proteína ya que cuenta con las propiedades nutricionales y sus características físicas le permite flotar en el agua por un período determinado, pero se desconoce el efecto en el crecimiento de los neonatos. El objetivo de este estudio consistió en 
comparar la adaptabilidad, el crecimiento y la sobrevivencia de neonatos de tortuga golfina (Lepidochelys olivacea) alimentados con una dieta comercial para tilapia y carne de corvina durante 35 días en cautiverio en el laboratorio húmedo del CURLP.

\section{MATERIALES Y METODOS}

\section{Área de estudio}

El proyecto se realizó en el laboratorio húmedo del Centro Regional Del Litoral Pacífico (CURLP) ubicado en la zona sur de Honduras a $158 \mathrm{~km}$ de Tegucigalpa, durante los meses de octubre a diciembre del 2018. Este cuenta con una precipitación acumulada anual de $1,287 \mathrm{~mm}$, una temperatura promedio diaria de $30.2^{\circ} \mathrm{C}$, siendo la temperatura máxima media anual de $36.3^{\circ} \mathrm{C}$ y la temperatura mínima media anual de $24.2^{\circ} \mathrm{C}$.

\section{Colecta y pesaje de individuos}

En este tiempo se mantuvo en cautiverio 12 especimenes en la fase de neonato de Lepidochelys olivacea que se manejaron bajo condiciones controladas. Los neonatos fueron adquiridos en el Campamento Tortuguero "Centro de Investigación de la Tortuga Marina" ubicada en la isla Boca de Rio Viejo a $36 \mathrm{~km}$ al sur del CURLP. Se utilizaron neonatos con peso promedio de $17 \mathrm{~g}$ y $66 \mathrm{~mm}$ de longitud que se trasladaron entre las 5 y 6 de la tarde con el propósito de no perjudicarlos por el efecto de la temperatura, durante el viaje se introdujeron en un recipiente plástico de $50 \mathrm{~L}$ de agua marina y se evitó generar condiciones de estrés en los animales.

\section{Instalación del ensayo}

Al llegar al laboratorio húmedo los neonatos fueron aclimatados en un recipiente más grande para mantenerlos a una temperatura estable y con aireación constante durante 120 minutos. Seguidamente fueron distribuidos en seis recipientes circulares con $0.22 \mathrm{~m} 3$ que fueron las unidades experimentales, estos se llenaron con un $50 \%$ de agua marina filtrada que tuvo la misma temperatura del agua a la que se trasladaron los animales. Sobre los recipientes para protegerlos de posibles depredadores y evitar contaminación externa, se instaló una malla de tela $2 \mathrm{~mm}$ de ojo de luz, y se incorporó aireación constante a cada recipiente. 


\section{Diseño de la investigación}

Se manejaron dos tratamientos que fueron el grupo de tortugas alimentadas con una dieta balanceada al $35 \%$ de proteína cruda para tilapia y el otro grupo que consumió carne de corvina. Cada tratamiento fue manejado por triplicado y se distribuyó aleatoriamente. En cada unidad experimental se manejó dos tortugas, siendo un total de 12 especimenes.

\section{Intervenciones}

Una vez instalado el experimento, los neonatos se alimentaron a las 72 horas después de haberlos llevado al CURLP, cuando fueron recolectados estos tenían 12 horas de nacidos y aun contaban con reservas alimenticias de su saco vitelino. Diariamente se verifico y se registró la sobrevivencia, así como los parámetros del agua (temperatura, oxígeno, $\mathrm{pH}$ y salinidad). Los recambios de agua se realizaron semanalmente en cada recipiente para eliminar desechos orgánicos producido por las tortugas y alimento no consumido con el propósito de mantener una buena calidad de agua. Para la manipulación se utilizó guantes de látex para prevenir enfermedades o cualquier contaminante que afectará a los neonatos.

Cada dos días se tomaron registros fotográficos observando cambios en su morfología durante su crecimiento. Semanalmente los neonatos fueron pesados usando una balanza digital y medidos con una cinta milimétrica. Mientras que el largo curvo del carapacho (LCC) que consistió en la medida desde la cabeza hasta el extremo de la cola y el ancho curvo del carapacho (ACC) fue la medición de la circunferencia del abdomen. Con esta información se analizó la adaptabilidad, crecimiento semanal y la sobrevivencia.

\section{Adaptabilidad}

Para evaluar la adaptabilidad se observó presuntivamente la actividad natatoria, la búsqueda del alimento, el desarrollo anatómico y la presencia de deformidades.

\section{Alimentación}

Diariamente fueron alimentados a saciedad, la ración fue fraccionada en tres porciones durante el día, en donde la última ración se aplicó con mayor volumen de alimento en las primeras horas de la noche, debido a que durante la noche se observó que los neonatos desarrollaron la mayor actividad. 


\section{Análisis estadístico}

Se utilizó la prueba estadística de hipótesis para comparar el crecimiento diario de las tortugas alimentadas con carne de corvina y alimento balanceado para tilapia al $35 \%$ proteína cruda, mediante la prueba t-student y la prueba de homogenidad de varianzas (prueba F) al 0.05 nivel de significancia Este mismo análisis se manejó para comparar los pesos finales de las tortugas. Se comparó la LCC semanal promedio de los dos grupos y se analizó por medio de la t-student. En la relación biométrica se manejó el análisis de relación para predecir los pesos en función del LCC, el peso en función del ACC en los dos tratamientos. Para evaluar la sobrevivencia se utilizó estadística descriptiva. En todos los análisis se manejó el software estadístico Infostat $\circledast 2018$.

\section{RESULTADOS}

\section{Adaptabilidad}

Se observó una adaptabilidad favorable en todos los neonatos que se manejaron en cautiverio. La actividad natatoria se presentó a partir de las 36 horas después de eclosionados, pero mostraron poca movilidad hasta llegar al cuarto día en donde tuvieron una respuesta favorable en la búsqueda del alimento. Durante los 35 días de estudio no se observó rechazo del alimento y el mayor consumo fue entre las 6 a 8 am y de 3 a 6 pm, siendo el período de mayor actividad entre las 3 a 7 pm.

Figura 1. Crecimiento de neonato en el laboratorio húmedo del CURLP durante 35 días de cautiverio, se observó cambio en la pigmentación del caparazón de color gris oscuro a verde olivo, el tamaño inicial fue de $70 \mathrm{~mm}$ llegando hasta los $92 \mathrm{~mm}$.
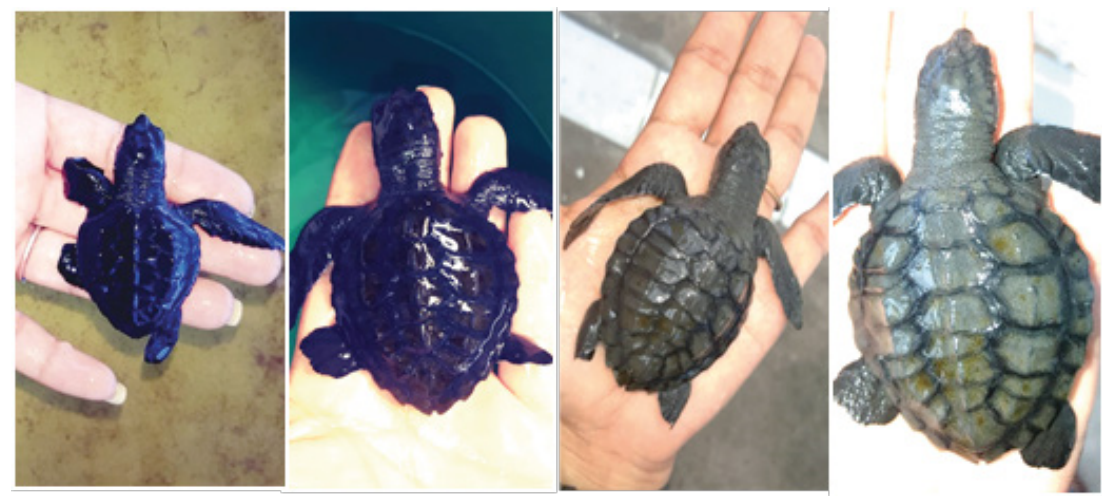

Fotografías: Kenia Rodríguez, Edgar Carranza. 
No se observó ningún tipo de deformidades en las extremidades, caparazón, cabeza y cola, las tortugas desarrollaron un crecimiento normal (Figura 1), cambiando la coloración de su cuerpo según crecían. Al nacer los neonatos eran de color gris oscuro y al llegar a la cuarta semana la pigmentación cambió a verde olivo en el caparazón y desarrollo una mayor dureza. Uno de los factores que favoreció la adaptabilidad de los neonatos fue el contar con un medio con las condiciones controladas, permitiendo manejar los parámetros ambientales que favorecieron la adaptabilidad.

\section{Crecimientos}

No se encontró diferencias estadísticas en la ganancia diaria de peso de las tortugas alimentadas con carne de corvina y alimento de tilapia $(P>0.999)$, a pesar de tener una ganancia diaria en promedio de $0.86 \mathrm{~g}$ para las tortugas alimentadas con carne de corvina y $0.46 \mathrm{~g}$ para los animales que consumieron alimento de tilapia. En la prueba de homogenidad de varianzas se obtuvieron diferencias estadísticas $(P<0.0002)$, siendo los crecimientos diarios de las tortugas que se alimentaron con carne de corvina los de mayor variabilidad. En los pesos finales no se encontraron diferencias concluyentes ( $P>0.1873$ ), en las tortugas alimentadas con carne de corvina el peso final fue de $37.83 \mathrm{~g}$ y $34.83 \mathrm{~g}$ para los neonatos alimentados con la dieta para tilapia (Figura 2). Ambas dietas favorecieron el crecimiento de los organismos.

En la comparación de la LCC del promedio semanal de los dos grupos, se encontró que los neonatos alimentados con carne de corvina fueron de mayor tamaño (Figura $3)$, pero estadísticamente estas diferencias no fueron concluyentes $(P>0.3652)$.

Figura 2. Comparación de pesos finales de neonatos de tortuga Lepidochelys olivacea alimentados con filete de corvina y alimento de tilapia al $35 \%$ de proteína cruda durante 35 días en cautiverio, en Choluteca, Honduras.

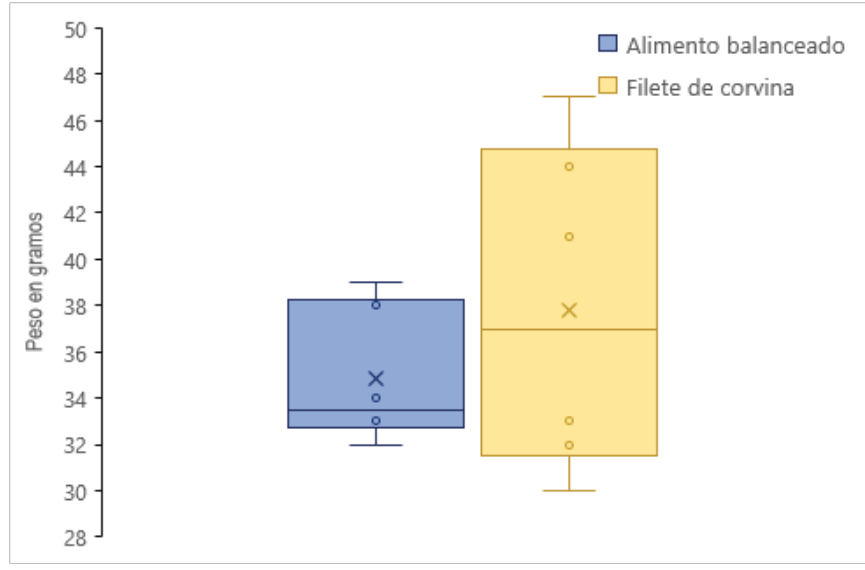


Figura 3. Comparación promedio del largo curvo del carapacho semanal en $\mathrm{mm}$ de neonatos de tortuga Lepidochelys olivacea alimentados con filete de corvina y alimento de tilapia al $35 \%$ de proteína cruda durante 35 días en cautiverio, en Choluteca, Honduras.

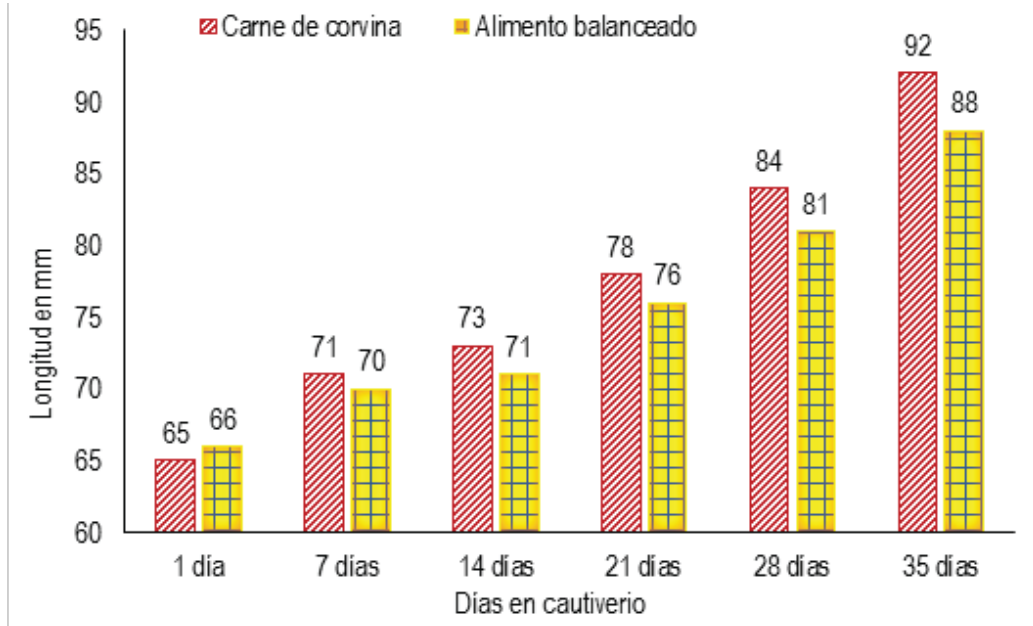

Fuente: elaboración propia

\section{Relación biométrica}

Las tortugas presentaron un aumento del peso y longitud semanalmente en las dos dietas aplicadas, sin embargo, se obtuvo una mayor variabilidad en los datos del grupo alimentado con la carne de corvina, el coeficiente de regresión se ajustó al modelo potencial para predecir el peso de la tortuga en función del LCC. Se observó una correlación media entre las dos variables (Figura 4). El coeficiente de regresión fue significativo $\left(R^{2}=0.8126\right)$, demostrando una relación entre las variables estudiadas.

En los animales alimentados con la dieta para tilapia se encontró un bajo coeficiente de determinación $\left(R^{2}=0.6185\right)$, lo que significa que hubo una poca relación entre el peso y la longitud de las tortugas. También se relacionó el peso con la ACC para predecir el peso del neonato en función de su anchura, y en los dos grupos de tortugas el coeficiente de determinación fue inferior al 0.6 , indicando que existe una baja correlación entre estas dos variables. 
Figura 4. Relación biométrica entre peso en $\mathrm{g}$ y longitud en $\mathrm{mm}$ del grupo de neonatos alimentados con carne de corvina durante 35 días en cautiverio.

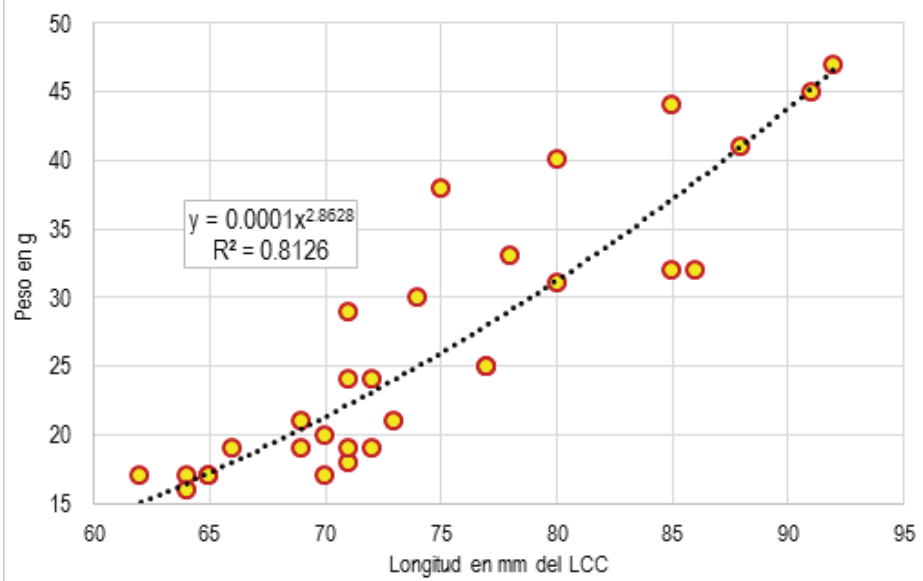

Fuente: elaboración propia

\section{Sobrevivencia}

La sobrevivencia fue del $100 \%$, no se registraron mortalidades durante las cinco semanas de estudio, en general los neonatos presentaron adaptación al medio de cautiverio y las condiciones de manejo favorecieron el desempeño de los animales al no observarse desarrollo de enfermedades o condiciones de estrés que puedan influir en la tasa de la sobrevivencia.

\section{DISCUSIÓN}

Los neonatos de la tortuga golfina presentaron una respuesta favorable en cautiverio, adaptándose a las condiciones que no fue su medio natural. Además, el buen desarrollo en los primeros 35 días fue influenciado positivamente por el manejo que recibió como los recambios periódicos del agua marina, la oxigenación constante del agua mantenido a $7 \mathrm{mg} / \mathrm{L}$ de oxígeno disuelto, y el tipo y calidad del alimento. Son pocos los estudios relacionados con el desempeño de las tortugas marinas en cautiverio, Kaufmann (1975) encontró que las tortugas Eretmochelvs imbricata y Caretta caretta empiezan a buscar alimento a partir del segundo día, información que se asemeja a lo observado en el experimento, en donde los neonatos tuvieron movilidad y búsqueda del alimento a partir del tercer día. Las tortugas marinas pueden adaptarse durante las primeras semanas de vida a crecer en condiciones de cautiverio. 
El alimento que fue ofrecido a los dos grupos estadísticamente no tuvo un impacto para mostrar diferencias en el tamaño y peso en los neonatos, debido a que el alimento de tilapia con el 35\% de proteína de cruda posiblemente contiene los requerimientos proteínicos y energéticos que demandan la tortuga golfina en las primeras semanas después de eclosionadas. Estos organismos son carnívoros (Colman, Sampaio, Weber, \& Comin, 2014), el contenido de proteína en su dieta debe ser alto y con buena digestibilidad, el alimento balanceado por ser una dieta formulada contiene estas propiedades que pueden favorecer en el crecimiento de las tortugas. Al ser adultos cambian sus hábitos e incorporan en su dieta el consumo de bentos y material vegetal (Bjorndal, 1997), con el uso de alimento balanceado en la etapa adulta la cantidad de proteína en la dieta podría reducirse e incrementar los niveles energéti$\cos$, ofreciendo los requerimientos nutricionales que necesita el animal.

A la adultez una tortuga llega a medir hasta $676 \mathrm{~mm}$ el largo del caparazón y lo alcanzan entre los 12 y 14 años de vida (Zug, Chaloupka, \& Balazs, 2006), esto es más de nueve veces el tamaño al nacer $(70 \mathrm{~mm})$. De igual manera inician pesando $0.15 \mathrm{~kg}$ y llegan hasta los $50 \mathrm{Kg}$ (Lara \& Rodríguez, 2014). La tortuga golfina es la especie que presenta el menor crecimiento en las tortugas marinas y se relaciona a la forma redondeada de su cuerpo (Kaufmann, 1975), mientras especies como Eretmochelys imbricata puede llegar a tener una tasa de crecimientos de $11.0 \mathrm{~g}$ por día en organismos de 11 meses de edad (Montero \& Peña, 1996), en la tortuga golfina se observó crecimientos entre 0.4 y $1.0 \mathrm{~g}$ por día. Se encontró el $100 \%$ de sobrevivencia en las tortugas alimentadas con las dos dietas, esto sugiere que la especie es tolerante a las condiciones en cautiverio, también la alta sobrevivencia puede deberse a que las tortugas tienen un metabolismo lento y una capacidad de adaptación al medio diferente al estado silvestre. Kaufman (1975) tuvo una alta tasa de sobrevivencia en grupos de 26 organismos de cuatro especies de tortugas marinas en cautiverio durante dos años, también (Montero \& Peña, 1996) reportaron una mortalidad del 0\% durante 183 días de estudio en 130 tortugas de la especie Eretmochelys imbricata alimentadas con carne de tilapia.

\section{CONCLUSIONES}

Las tortugas lograron adaptarse al manejo en cautiverio, no presentaron deformidades 0 enfermedades durante los 35 días de estudio. Esta adaptación puede ser influenciado por las condiciones de manejo que generó el bienestar animal en los neonatos de los grupos de tortuga alimentados con las dos dietas. En los crecimien- 
tos no se encontró diferencias estadísticas en los pesos ganados semanalmente $(P>0.999)$. En los pesos finales que fueron $37.83 \mathrm{~g}$ para los neonatos alimentados con carne de corvina y $34.83 \mathrm{~g}$ para los que consumieron la dieta balanceada de tilapia no se encontraron resultados concluyentes $(P>0.1873)$, a pesar de que los promedios finales fueron diferentes estos no lograron ser significativos.

En la comparación del LCC no se encontraron diferencias entre los dos grupos alimentados con las dos dietas ( $P>0.3652$ ), el alimento no influyó en el peso y tamaño de las tortugas. La dieta balanceada de tilapia al $35 \%$ de proteína cruda puede ser manejada como una alternativa a bajo costo y accesible para alimentar a los neonatos de tortuga hasta que alcancen una edad que les permita lograr una mejor sobrevivencia al ser liberados al medio natural. La sobrevivencia obtenida fue del $100 \%$ en todos los neonatos sometidos al estudio, las tortugas debido a su metabolismo tienen capacidad de adaptación diferente al medio natural, el tipo de alimento que se suministró en los animales no influyó en la sobrevivencia, y es más relacionado con las condiciones de manejo que favorecieron el bienestar animal y la bioseguridad.

\section{REFERENCIAS BIBLIOGRÁFICAS}

Aggarwal, R. K., Velavan, T. P., Hendre, P., \& Shanker, K. (2004). Development and characterization of novel microsatellite markers from the olive ridley sea turtle (Lepidochelys olivacea). Molecular Ecology Notes, 2-4. doi:10.1046/j.1471-8286.2003.00574.x

Bjorndal, K. A. (1997). Foraging ecology and nutrition of sea turtles. En P. L. Lutz, \& J. A. Musick, The biology of sea turtles (págs. 199-231). Boca Ratón, Florida, USA: CRC Press.

Camacho, L., Amorocho, D., Mejía, L., Palacio, J., \& Rondón, F. (2008). Caracterización genética de la colonia reproductiva de la tortuga marina golfina -Lepidochelys olivacea-en el parque nacioal natural Gorgona (Pacífico colombiano) a partir de secuencias de ADN mitocondrial. Boletín de Investigaciones Marinas y Costeras, 37(1), 77-92. Recuperado el octubre de 2018, de http://www.scielo.org.co/pdf/mar/v37n1/v37n1 a05.pdf

Casas , G., \& Gómez Aguirre, S. (1980). Contribución al conocimiento de los hábitos alimenticios de Lepidochelys olivacea y Chelonia mydas agassizi (Reptilia, Cheloniidae) en el Pacífico Mexicano. S. Paulo, 29(2), 87-89.

Castro González , I., Miranda Becerra, D., \& Pérez Gil, R. (2010). Tortuga golfina (Lepidochelis olivacea): Análisis nutrimental del huevo no eclosionado para su posible inclusión en la dieta humana. XII Congreso Nacional de Ciencia y Técnología de Alimentos (págs. 171-181). Guanajuato: Universidad de Guanajuato.

Colman, L., Sampaio, C. L., Weber, M. I., \& Comin, J. (2014). Diet of Olive Ridley Sea Turtles, Lepidochelys olivacea, in the Waters of Sergipe, Brazil. Chelonian Conservation and Biology, 13(2), 266-271. doi:http://dx.doi.org/10.2744/CCB-1061.1

IAC/CIT. (2007). Convención Interamericana para la Protección y Conservación de las Tortugas 
Marinas. Recuperado el 04 de enero de 2019, de Tortugas marinas: una lucha por la supervivencia: http://www.iacseaturtle.org/docs/publicaciones/12-Amenazas-FinalCOLOR-ESP.pdf

IUCN. (29 de septiembre de 2011). International Union for Conservation of Nature. Recuperado el 04 de enero de 2019, de Especialistas identifican a las poblaciones de tortugas marinas más amenazadas del mundo: https://www.iucn.org/es/content/especialistas-identifican-las- poblaciones-de-tortugas-marinas-m\%C3\%A1s-amenazadas -del-mundo

Kaufmann, R. (1975). Observaciones sobre el crecimiento en tortugas marinas en cuatividad. (I. C.-A. Betin", Ed.) Caldasia, 11(53), 139-150. Obtenido de https://revistas.unal.edu.co/index.php/cal/article/view/34310/34492

Lara, M. M., \& Rodríguez, C. M. (octubre de 2014). Conociendo la tortuga golfina Lepidochelys olivacea (Eschscholtz, 1929). (C. E. Faggioli, Ed.) Bioma, 2(24), 9-17. Obtenido de http://virtual.ues.edu.sv/bioma/

Marquez, R. (2013). La ciencia para todos. (I. L. Educativa, Ed.) Recuperado el 08 de enero de 2019, de Las tortugas marinas y nuestro tiempo: http://bibliotecadigital.ilce.edu.mx/sites/ciencia/volumen3/ciencia3/144/html/sec_7.htm

Mi Ambiente. (10 de Noviembre de 2018). Secretaría de Recursos Naturales y Ambiente (Honduras). Recuperado el enero de 2019, de MiAmbiente+ y CVC-GOLF Perpetúan y garantizan tortugas marinas en Golfo de Fonseca: http://www.miambiente.gob.hn/blog/view/miambiente-y-cvc-golfperpetuan-y-garantizan-tortugas-marinas-en-golfo-de- fonseca

Montero, W. G., \& Peña, J. C. (1996). Crecimiento, conversión de alimento y mortalidad de Eretmochelys imbricata (Reptilia: Chelonidae) en estanques artificiales en Costa Rica. Revista de biología trópical, 44(2), 847-851.

Pelegrín, E., Álvarez, S., Fraga, I., \& Galindo, J. (2003). Requerimientos de proteína en subjuveniles de tortuga carey (Eretmochelys imbricata). CIVA, 978-985. Obtenido de https://www.oceandocs.org/bitstream/handle/1834/3710/Elda\%20Pelegr\%C3\% ADn\%20Morales\%5B1\%5D.\%20CIVA\%201.pdf?sequence=1\&isAllowed=y

PNUD. (2019). PNUD: Honduras. Obtenido de Científicos y pobladores del sur unidos por la tortuga golfina: http://www.hn.undp.org/content/honduras/es/home/ourwork/environmen tandenergy/successstories/tortuga_golfina.html

Wilson, E., Miller, K., Allison, D., \& Magliocca, M. (2006). Oceana. Obtenido de Why healthy oceans need sea turtles: the importance of sea turtles to maryne ecosystems: http://usa.oceana.org/sites/default/files/reports/Why_Healthy_Oceans_Need_Sea_Turtles.pdf

Zug, G. R., Chaloupka, M., \& Balazs, G. H. (2006). Age and growth in olive ridley seaturties \{Lepidochelys olivácea) from the North-central Pacific: a skeleto chronological analysis. Marine Ecology, 27, 263-270. doi:doi:10.1111/j.1439-0485.2006.00109.x 